BMJ Open Sport \& Exercise Medicine

\title{
10-year follow-up after standardised treatment for Achilles tendinopathy
}

Finn Johannsen, ${ }^{1,2}$ Signe Jensen, ${ }^{2}$ Eva Wetke ${ }^{3}$

To cite: Johannsen F, Jensen S, Wetke E. 10-year follow-up after standardised treatment for Achilles tendinopathy. BMJ Open Sport \& Exercise Medicine 2018;0:e000415. doi:10.1136/ bmjsem-2018-000415

Accepted 3 September 2018
Check for updates

\section{(c) Author(s) (or their} employer(s)) 2018. Re-use permitted under CC BY-NC. No commercial re-use. See rights and permissions. Published by BMJ.

${ }^{1}$ Institute of Sports Medicine Copenhagen, Bispebjerg Hospital, Copenhagen, Denmark ${ }^{2}$ Furesø-reumatologerne, Farum, Denmark

${ }^{3}$ Department of Orthopedic Surgery, Næstved, Denmark

Correspondence to Finn Johannsen; F.E. Johannsen@dadlnet.dk

\section{ABSTRACT}

Background Achilles tendinopathy is a common and often long-lasting injury. We present a 10-year follow-up on a pragmatic study on Achilles tendinopathy treated with controlled exercises supplemented with corticosteroid injections if necessary in order to continue training. Methods All patients who completed the original study ( $n=93$ ) were invited for a 10-year follow-up. $83 \%$ participated. Patients were evaluated with ultrasound scanning $(n=58)$ and with a questionnaire $(n=77)$ using the same outcome measures as in the primary study. The 10year overall outcome on a 4-point scale (excellent, good, fair, poor), other treatments and adverse event and present activity level were recorded.

Results Excellent outcome was reported in 63\% and good outcome in $27 \%$. $76 \%$ reported an activity level at $75 \%-100 \%$ of preinjury level. The average Victorian Institute of Sports Assessment-Achilles score for all patients was 84 (SD 19). 16\% had surgery. Three ruptures occurred 5-8 years after the primary study. The improvement from entry to 6 months in the primary study was maintained until 10-year follow-up. Insertional tendinopathy did not differ from mid-substance tendinopathy in any outcome measure (short term and long term). We encountered no prognostic markers on ultrasound for the long-term outcome; however, present heterogeneity and increased flow resemble present pain. Thickened tendons seem to maintain their thickness despite improvement of symptoms.

Conclusion One to two corticosteroid injections are a safe and effective supplement to controlled exercises in the treatment of Achilles tendon pain with no signs of deterioration in the very long term. Mid-substance and insertional tendinopathies benefit equally from this treatment.

\section{INTRODUCTION}

Achilles tendinopathy (AT) is a common and often long-lasting injury. There are many recommended conservative treatment modalities for AT, meaning no one is really excellent. Eccentric exercises are often recommended, ${ }^{1}$ with reported improvement in $60 \%{ }^{23}$ to $90 \% .^{4-6}$ Malliaras et al found that there was no clear evidence that favoured eccentric exercises over eccentric/concentric exercises. Beyer $e t a l$ found that heavy slow resistance exercise three times a week was as good as daily eccentric exercises. ${ }^{8}$ Other
How might it impact on clinical practice in the near future?

One to two corticosteroid injections are a good and safe supplement to controlled exercises in patients with Achilles tendinopathy.

- Mid-substance tendinopathy and enthesopathy can be treated with the same treatment protocol with equal effect.

- Ultrasound scanning cannot predict long-term outcome in patients with Achilles tendinopathy.

- Present heterogeneity or increased flow correlate with present pain in Achilles tendinopathy.

studies have shown the effect of stretching exercises. ${ }^{69}$ Fredberg et $a l^{10}$ found good shortterm effect of peritendinous injection of corticosteroid (CS), but an aggressive rehabilitation resulted in a relapse after 6 months. ${ }^{10}$ These randomised clinical efficacy trials have been performed by highly motivated physiotherapists and investigators seeking high compliance on selected motivated patients agreeing in supervised training, and it is doubtful that the same success rate can be achieved on all patients. ${ }^{11}$

Some studies have looked at the very longterm follow-up (5-8 years). ${ }^{12-14}$ At 5 years Silbernagel et $a l^{12}$ reported $65 \%$ were symptom-free, ${ }^{12}$ whereas van der Plas et al ${ }^{13}$ found only $39.7 \%$ without symptoms, ${ }^{13}$ and Paavola et $a l^{14}$ reported $59 \%$ symptom-free after 8 years. $^{14}$

In 2004/2005 we performed a pragmatic study on all consecutive patients with AT being referred to one of two outpatient rheumatology clinics in Copenhagen, Denmark during a 10-month period. ${ }^{15}$ All patients were treated with the same treatment regimen regardless of the location of tendinopathy: reduced running and jumping activities, concentric/eccentric exercises, calf stretching, and if training was impossible due to too severe pain supplemented with one to three ultrasound-guided CS injections (1 $\mathrm{mL}$ Lidocaine $10 \mathrm{mg} / \mathrm{mL}$ and $1 \mathrm{~mL}$ methylprednisolone acetate $40 \mathrm{mg} / \mathrm{mL}$ ) in order to continue the training. Twenty-four succeeded with training alone, 54 had one CS injection, 
13 had two CS injections and 2 had three CS injections. At follow-up after 6 months, 94\% had improved and $76 \%$ were symptom-free or had only slight symptoms. Bakkegaard et $a l^{16}$ have described the ultrasound scanning (US) findings in that study and found that heterogeneity, defined as clearly demarcated hypoechoic or hyperechoic areas within the tendon at the initial examination, was found to be a prognostic marker for poor clinical outcome at 6 months.

The objectives of this study were to investigate the very long-term result (10 years) of the patients included in our previous study and to investigate if CS injections influenced this outcome and also if US is useful for the very long-term prognosis.

\section{MATERIALS AND METHODS}

In the original study 113 consecutive patients were clinically diagnosed with AT. US confirmed the diagnosis. Eight juvenile and two pre-existing ruptures were excluded. Ten patients dropped out for various reasons, resulting in 93 patients completing the study. Sixty-eight were active in sports preinjury with a mean of 5.3 hours of activity per week, but the activity level did not influence the outcome. Seventy-five with mid-substance tendinopathy and 18 with insertional tendinopathy improved equally from the treatment.

All patients who completed the original study $(n=93)$ were invited 10 years after the last person finished the primary study, meaning that patients were invited 10 years and 0 month to 10 years and 10 months after finishing the primary study. Patients were invited to a physical examination including US and/or a questionnaire.

\section{Patient involvement}

Patients were not involved in setting the research agenda.

All patients were invited by phone or letter to participate in this follow-up. Seventy-seven $(83 \%)$ participated, 19 answered our questionnaire by phone $(n=14)$ or letter $(\mathrm{n}=5)$, and 58 showed up for examination including US (figure 1).

An independent investigator (SJ) evaluated the patients' symptoms and function. We registered the weight, activity level in per cent compared with before the injury, change of sport and the reason, and global assessment of function over the past 10 years on a 4 -grade scale-1: excellent, no physical limitations; 2: good, slight physical limitation; 3: fair, considerable physical limitations; and 4: poor, severe physical limitation, and whether the Achilles tendon was the reason for these limitations. They were also asked if they had experienced Achilles tendon rupture and if they had sought other treatment and received any CS injections after our study ended.

Symptoms were evaluated as in the primary study using a $100 \mathrm{~mm}$ Visual Analogue Scale. We recorded pain during function after doing 20 one-legged heel rises, pain at palpation by pinching the tendon gently between two fingers, and self reported morning pain. In the primary study, we were not aware of a quantitative pain

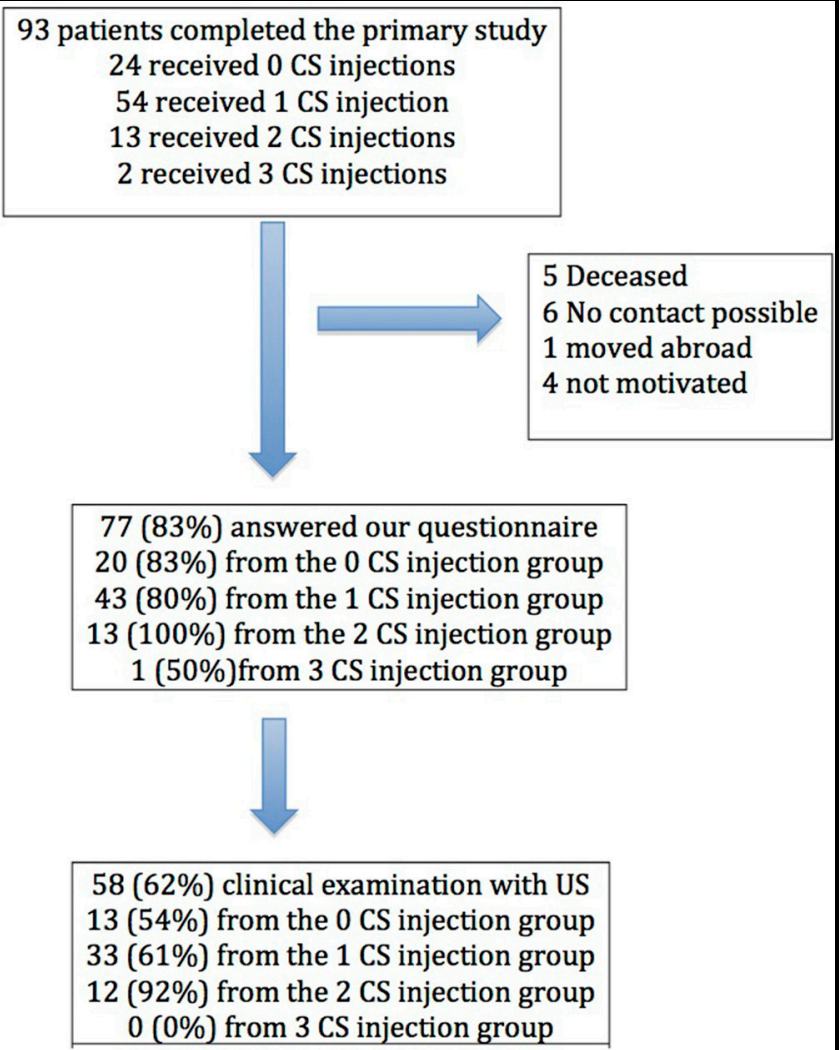

Figure 1 Patients' flow chart. CS, corticosteroid; US, ultrasound scanning.

and function score, but for the 10-year follow-up we used the 'Victorian Institute of Sports Assessment - Achilles' (VISA-A), which recently was validated and reliability-tested in Danish-speaking patients with tendinopathy. ${ }^{17}$ These outcome measures were only assessed on patients showing up for clinical examination or by telephone contact for proper instruction.

The clinical examination consisted of US by one of the investigators of the primary study (FJ) using the same standards and definitions. The US machine used was Hi Vision Avius (Hitachi Medical Systems UK) with an $18-5 \mathrm{MHz}$ linear array transducer. US diagnostics were performed with the patient lying prone face down with the feet hanging free over the edge and the ankle at approximately $90^{\circ}$ dorsiflexion without tension to the tendon. The tendon was scanned longitudinally and transversally. The thickness was measured at the thickest part of the tendon on a transverse scan, which we have shown to be reliable. ${ }^{18}$ A colour Doppler was used to evaluate the flow according to the Newman Grading Scale (0-3) after the Doppler was calibrated to zero pixels at an asymptomatic area on the gastrocnemius part of the tendon. We were also looking for hypoechoic and hyperechoic changes within the tendon and for bursitis.

\section{Statistics}

Association between increased intratendinous flow, heterogeneity, insertional or intratendinous affection 
and tendon thickness both at the initial examination and at 10-year follow-up compared with 10-year outcome measured by VISA-A score was tested using non-paired t-test for dichotomous variables and by Pearson correlation for continuous variables.

Association between US measures initially and after 10 years was tested with Fisher's exact test for dichotomous variables and Pearson correlation for Gaussian distributed continuous variables, otherwise Spearman correlation.

Intergroup differences $(0,1$ or 2 CS injections) in the 10-year outcome measures were calculated using one-way analysis of variance. For intragroup differences we used paired t-test.

\section{RESULTS}

Overall $63 \%$ reported excellent outcome with no physical limitation in the past 10 years. A further $27 \%$ reported only slight limitation. Overall $76 \%$ reported an activity level at $75 \%-100 \%$ of preinjury level, and the average VISA-A score for all patients was 84 (SD
19), and $64 \%$ were above 90 . Twenty-two per cent had changed sport due to Achilles tendon problems.

Sixteen per cent had surgery, $10 \%$ for mid-substance tendinopathy and $43 \%$ for insertional tendinopathy. Three tendon ruptures were reported, one in each group ( 0,1 and 2 CS injections). The patient in the 0 CS group had received six CS injections from another doctor before rupture. The other patients sustained their ruptures 5-8 years after the CS injection in our study and had had no other injections.

Patient characteristics at 10-year follow-up are presented in table 1 , divided into the three primary treatment groups.

The single patient with three CS injections is not included in the statistic analysis due to the small number. For comparison the average activity level compared with preinjury level of patients receiving, respectively, zero, one and two CS injections was $85 \%, 83 \%$ and $74 \%$ (no intergroup difference), and the average VISA-A scores were, respectively, 87 (SD 17), 85 (SD 16) and 76 (SD 27), with no intergroup differences.

Table 1 Patients' characteristics at 10-year follow-up divided into the three primary treatment groups

\begin{tabular}{|c|c|c|c|}
\hline & $0 \mathrm{CS}$ injection & $1 \mathrm{CS}$ injection & $2 \mathrm{CS}$ injections \\
\hline$n$ & 20 & 43 & 13 \\
\hline Female & 4 & 24 & 7 \\
\hline Age (SD) & $56(10.8)$ & $60(11.5)$ & $58(9.4)$ \\
\hline Weight (SD) & $83.9(13.7)$ & $76.0(14.7)$ & $83.2(10.2)$ \\
\hline Change of sport during 10 years & 8 & 20 & 6 \\
\hline Due to Achilles tendon & 4 & 9 & 4 \\
\hline \multicolumn{4}{|l|}{ Other treatment past 10 years } \\
\hline Operation & 1 & 8 & 3 \\
\hline CS injection & 3 & 1 & 2 \\
\hline Other & 1 & 4 & 3 \\
\hline Physical function past 10 years & (19 answers) & (43 answers) & (13 answers) \\
\hline Excellent: no limitations & $13(68 \%)$ & $29(67 \%)$ & $5(38 \%)$ \\
\hline Good: slight limitation & $5(26 \%)$ & $12(28 \%)$ & $3(23 \%)$ \\
\hline Fair: large physical limitation & $0(0 \%)$ & $2(5 \%)$ & $5(38 \%)$ \\
\hline Poor: severe physical limitation & $1(5 \%)$ & $0(0 \%)$ & $0(0 \%)$ \\
\hline Activity level compared with preinjury level & (19 answers) & (43 answers) & (13 answers) \\
\hline $0 \%-24 \%$ & $1(5 \%)$ & $2(5 \%)$ & $1(8 \%)$ \\
\hline $25 \%-49 \%$ & $1(5 \%)$ & $3(7 \%)$ & $1(8 \%)$ \\
\hline $50 \%-74 \%$ & $2(11 \%)$ & $3(7 \%)$ & $4(31 \%)$ \\
\hline $75 \%-100 \%$ & $15(79 \%)$ & $35(81 \%)$ & $7(54 \%)$ \\
\hline VISA-A score & (19 answers) & (43 answers) & (13 answers) \\
\hline $0-49$ & $1(5 \%)$ & $1(2 \%)$ & $4(31 \%)$ \\
\hline $50-74$ & $1(5 \%)$ & $9(21 \%)$ & $0(0 \%)$ \\
\hline $75-89$ & $4(21 \%)$ & $6(14 \%)$ & $1(8 \%)$ \\
\hline $90-100$ & 13 (68\%) & 27 (63\%) & $8(62 \%)$ \\
\hline
\end{tabular}

CS, corticosteroid; VISA-A, Victorian Institute of Sports Assessment-Achilles. 

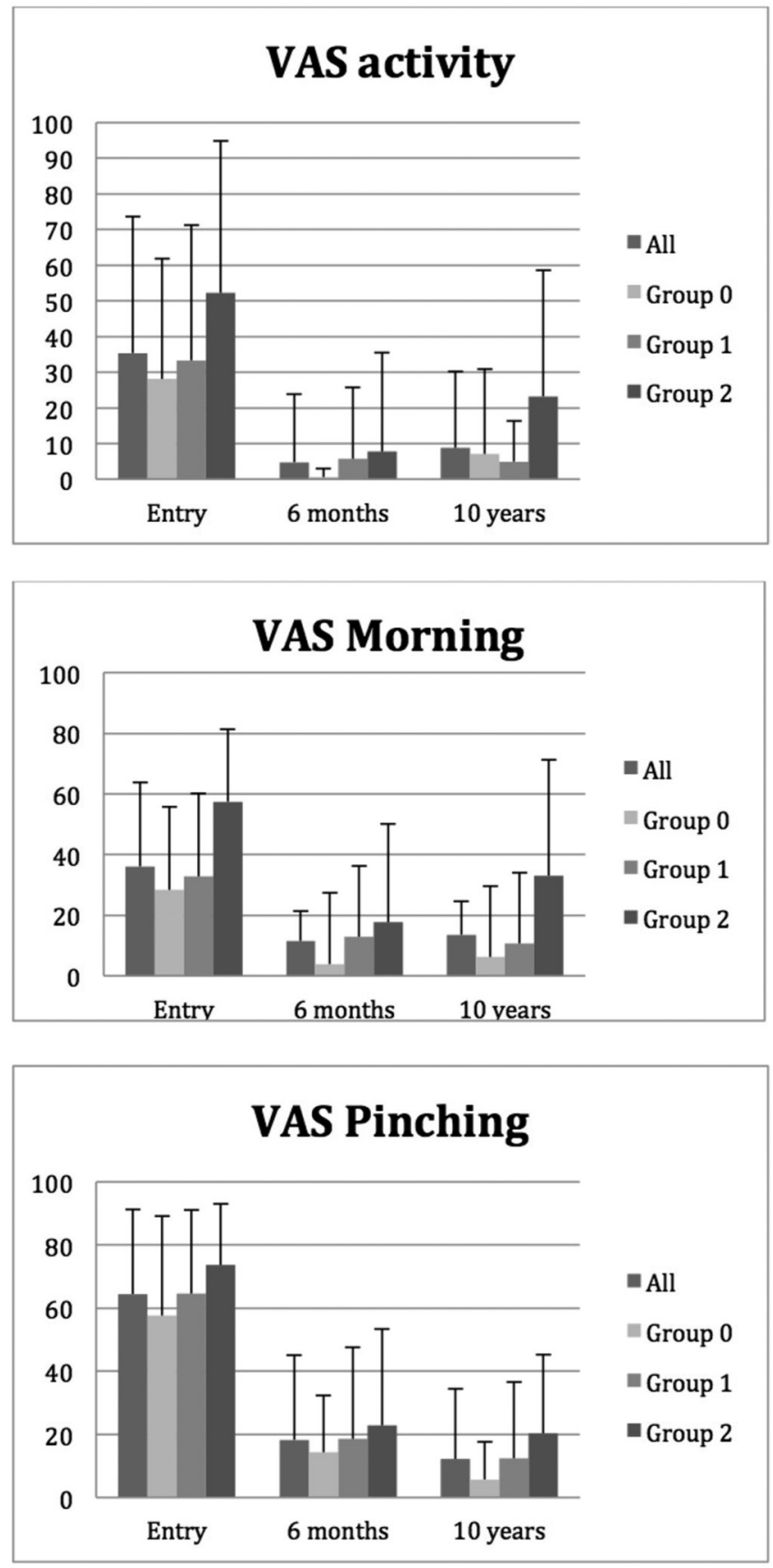

Figure 2 Pain during activity, in the morning and by pinching is illustrated at entry, after 6 months and after 10 years. I bars illustrate SD. Groups 0, 1 and 2 had zero, one or two corticosteroid injections, respectively, in the primary study. All groups improved significantly from entry to 6 months and entry to 10 years, without significant changes from 6 months to 10 years. Group 2 was significantly different from the other groups at 10-year follow-up: VAS activity $p=0.022$, VAS morning $p=0.016$ compared with the other groups (one-way analysis of variance). CS, corticosteroid; VAS, Visual Analogue Scale.

\section{Pain outcome measures}

In figure 2 the pain outcome measures are presented at entry, at 6 months and at 10-year follow-up. All outcome measures improved significantly in the primary study from entry to 6 months for the total group of patients and for each subgroup of patients receiving zero, one or two CS injections. This improvement remained significant until 10-year follow-up for the total group as well as for each subgroup. However, comparing each subgroup at 10 years, the subgroup of patients receiving two CS injections scored significantly higher than the other treatment groups in pain during function $(\mathrm{p}<0.0001)$ and morning pain ( $p=0.013)$, but without significant difference in pain by pinching.

\section{Insertional and mid-substance tendinopathies}

Fourteen insertional and 63 mid-substance tendinopathies (defined as the thickest part of the tendon on US) were included in the follow-up and they had equal long-term outcome. VISA-A score for all patients with insertional tendinopathy was 90 (SD 10), for tendinopathy 1-2 cm above insertion was 87 (SD 17) and for mid-substance tendinopathy 80 (SD 21), with no intergroup differences. These scores included the 12 operated patients ( 6 insertional, 1 just above insertion, 5 mid-substance). Excluding the operated patients the VISA-A score for insertional tendinopathy was 90 (SD 6 ), tendinopathy $1-2 \mathrm{~cm}$ above insertion was 86 (SD 18) and for mid-substance tendinopathy 82 (SD 20), with no intergroup differences. These good results for insertional tendinopathy might be too optimistic as a big proportion (6/14) of these patients underwent surgery.

\section{Ultrasound scanning}

Figure 3 illustrates the change in thickness of the Achilles tendon. For the total group a significant decrease in thickness was found from entry to 6 months $(\mathrm{p}<0.0001)$ and from entry to 10 years $(p=0.004)$; however, from 6 months to 10 years, thickness increased significantly $(p=0.001)$. Only the two subgroups receiving CS injections showed a significant decrease from entry to 6 months $(\mathrm{p}<0.0001$ and $\mathrm{p}=0.011$, respectively $)$, and the group receiving two CS injections showed significant worsening from 6 months to 10 years $(p=0.012)$, and the group receiving one $\mathrm{CS}$ injection showed tendency to worsening $(\mathrm{p}=0.07)$.

Neither the tendon thickness at entry nor the tendon thickness at 10-year follow-up correlated with the 10-year VISA-A score (Spearman test), but as illustrated in figure 4 the thickness at entry and at 10-year follow-up correlated ( $r=0.56, \mathrm{p}<0.0001$, Pearson).

Heterogeneity or increased flow at entry did not predict poor outcome measured by VISA-A score, although increased flow grades 2-3 at 10-year follow-up had significantly poorer VISA-A score compared with grades $0-1$ flow $(\mathrm{p}=0.0015)$. Also present heterogeneity showed a strong tendency to lower VISA-A score $(p=0.070)$. We found no correlation in neither flow nor heterogeneity from entry to 10-year follow-up, but this could be explained by differences in the US machines and the development of more sensitive Doppler function in the newer machines. 


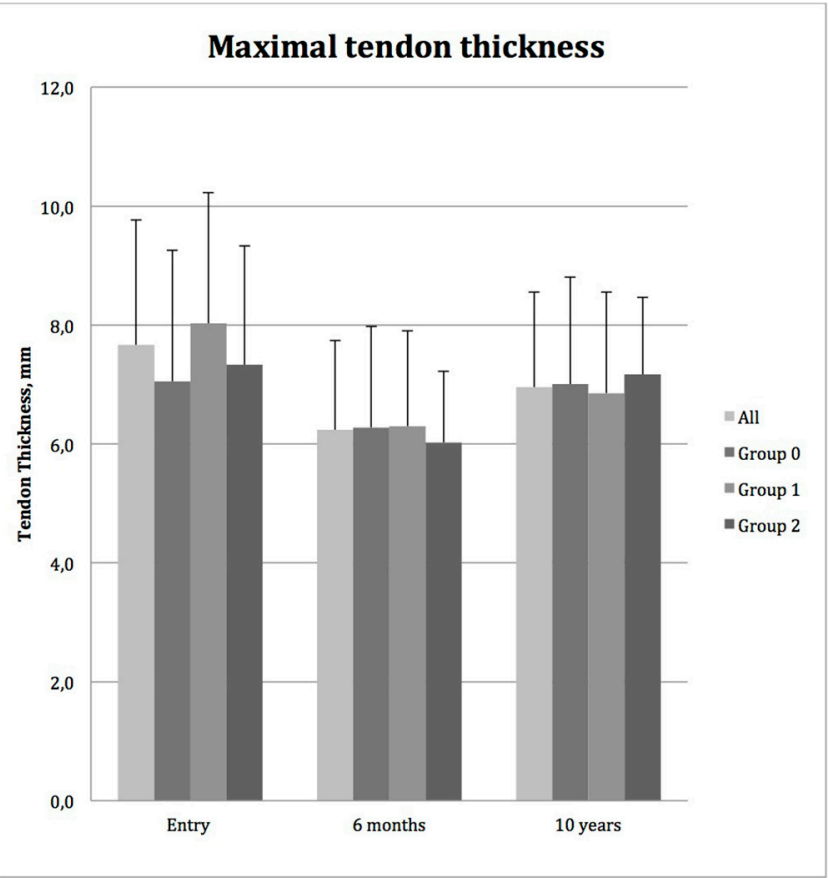

Figure 3 The maximal tendon thickness measured by ultrasound scanning at entry, 6 months and 10 years. I bars illustrate SD. Groups 0, 1 and 2 had zero, one or two corticosteroid injections, respectively, in the primary study. Thickness decreased significantly from entry to 6 months for the total group and groups 1 and 2, and increased significantly from 6 months to 10 years for the total group and group 2.

\section{DISCUSSION}

\section{Corticosteroid in AT}

We have described the long-term effect of a treatment regimen for AT consisting of reduced loading, slowly progressive concentric and eccentric strengthening exercises, stretching exercises, supplemented with one to three CS injections if patients were unable to commence

\section{tendon thickness correlation}

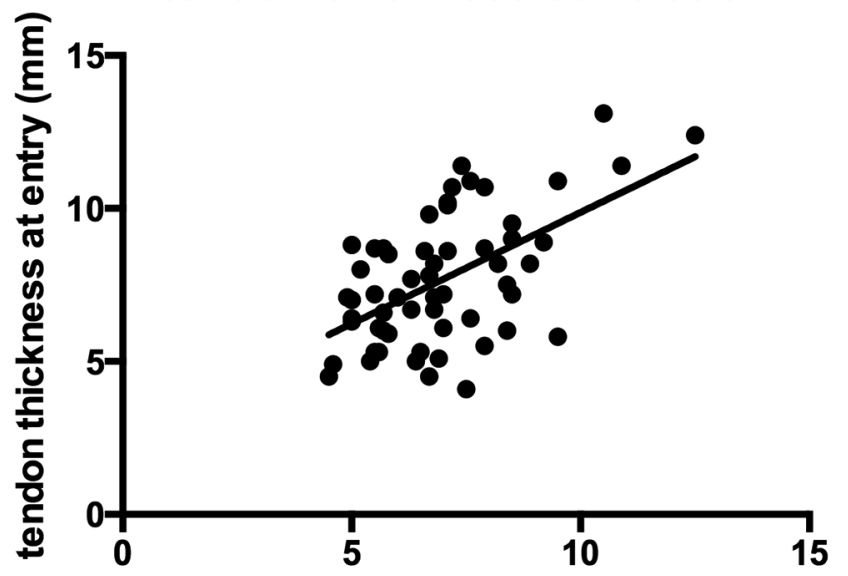

tendon thickness at 10 years follow up $(\mathrm{mm})$

Figure 4 Correlation between the primary measured tendon thickness and the thickness measured at 10-year follow-up. $r=0.56, p<0.0001$. or progress in exercise. We have previously described the short-medium term (6 months) effectiveness of this treatment and found that $94 \%$ improved, and $77 \%$ with good or excellent effect. ${ }^{15}$ In the present study $90 \%$ reported excellent or good results after 10 years. This is in contrast to a study by Fredberg $e t a l,{ }^{10}$ who describe a poor outcome after CS injection after 6 months. ${ }^{10}$ A meta-analysis on C.S injections for tendinopathy in general also confirms poor outcome in the long term. ${ }^{19}$ This could be due to the fact that most randomised controlled trial (RCT) studies on CS injections mainly use a single CS injection as monotherapy, whereas we used one to three CS injections as a supplement and only if necessary in order to proceed with a cautious and slowly progressive training protocol. Therefore it seems that CS might reduce warning signals and lead to too quick training progression, if training is not controlled by a cautious protocol.

Our very long-term results are comparable with other long-term follow-up studies. ${ }^{12-14}$ Paavola et al ${ }^{14}$ described an observational 8-year follow-up study on patients treated for AT with many different kinds of treatments including one to three CS injections and found $94 \%$ had good or excellent results. ${ }^{14}$ Silbernagel et $a l^{12}$ did a 5-year follow-up on an efficacy RCT including specific eccentric training for AT and reported $65 \%$ were symptom-free, ${ }^{12}$ whereas van der Plas $e t a l^{13}$ on a similar study group found only $39.7 \%$ without symptoms at 5 -year follow-up. ${ }^{13}$ In the present study $63 \%$ were symptom-free.

All outcome measures in our primary study were significantly improved, and this was maintained at 10-year follow-up. However the group of patients receiving two C.S injections had significantly poorer outcome at 10 years in morning pain and pain during function, although not in VISA-A score compared with the other groups. Also we found that only this group showed a significant increase in tendon thickness from 6 months to 10 years. A higher proportion of patients receiving CS underwent surgery compared with the group without an injection.

This might be due to a deleterious effect of CS reducing collagen synthesis, ${ }^{20}$ but can most probably be explained by the fact that patients who were offered CS injections in our study had more severe AT.

\section{Surgery and tendon ruptures}

A bias to any long-term follow-up is that during a period of 5-10 years patients may have sought other treatments. Paavola et $a l^{14}$ reported $29 \%$ being operated Silbernagel et al $0 \%^{12}$ and van der Plas et al $13 \% .^{13}$ In the present study $16 \%$ had surgery. The reasons for these differences could be the selection of patients in the different studies and different inclusion criteria. In our study we included consecutive patients, including patients with insertional tendinopathy.

Tendon ruptures after CS injections are mainly reported in case studies, but in prospective studies only one rupture has been reported. ${ }^{101921}$ In very longterm follow-up studies, one rupture was described by Paavola et $a l^{14}$ in a patient playing soccer 1 week after a 
CS injection. ${ }^{14}$ In our study three ruptures were registered over the 10-year period, one of these obtained after aggressive CS injection therapy with six injections in another medical clinic. However, the remaining two ruptures occurred 5-8 years after CS injections. Also two patients in the present study had had pre-existing ruptures at entry without any CS injections and were excluded from our study. We conclude that tendinopathy might lead to rupture, and consider it possible that more than three injections might increase the risk, especially if combined with aggressive rehabilitation. ${ }^{10}$ Based on our study we believe that one to three injections combined with reduced loading and controlled exercises is safe, and according to Paavola et $a l^{14}$ and our primary and present study with good short-term and long-term outcomes.

\section{Insertional and mid-substance tendinopathies}

In our study we included both mid-substance and insertional tendinopathies, and in the primary study we found equal effect, with $77 \%$ being satisfied with our treatment regimen. However the classic painful eccentric training proved to improve $60 \%-90 \%$ of patients with mid-substance tendinopathy, ${ }^{2-6}$ and only had a good effect in $32 \%$ of patients with insertional tendinopathy. ${ }^{5}$ Our training regimen started with exercises without dorsiflexion and slowly progressed to dorsiflexed exercises not aggravating pain. In a non-controlled pilot study on patients with insertional AT, $67 \%$ of patients were satisfied after performing 12 weeks of eccentric exercises without dorsiflexion. ${ }^{22}$ Many studies argue that mid-substance and insertional tendinopathies have different pathology, and therefore exclude insertional tendinopathy in treatment studies $^{2-46}$; however, the pathology seems similar. ${ }^{23}$ AT might also change over time from mid-substance to insertional or vice versa, and Paavola et al describe how three patients in their study changed from mid-substance to insertional tendinopathy during the follow-up period. ${ }^{14}$ We did not see that change in our study, but the long-term result was just as good for the insertional tendinopathy as for mid-substance tendinopathy.

\section{Ultrasound scanning}

In our primary study population we found that heterogeneity at entry was a prognostic factor for poor outcome, ${ }^{16}$ although tendon thickness and Doppler activity at entry could not be used as prognostic markers. ${ }^{16}$ In this longterm follow-up, no US changes at entry were found useful as prognostic marker in the very long term.

Tendon thickness, increased flow and hypoechogenicity were found to be associated with pain at any given time point in our primary study population. ${ }^{16}$ Reiter $e t$ $a l^{24}$ found a positive relationship between the presence of neovascularisation and pain and restricted function on 20 patients, ${ }^{24}$ but De Jonge $e t a l^{25}$ could not confirm this finding in a bigger study comprising 556 paired measurements but had dichotomised the flow from Ohberg grades $0-4 .^{26}$
Boesen et $a l^{27}$ have shown that neovascularisation might be a physiological response to activity. ${ }^{27}$ In our study grade 1 flow did not differ in pain and function measured by VISA-A from grade 0 , but grades 2-3 had significant lower VISA-A than grade 0 . Therefore some degree of flow might be physiological, whereas higher grades of flow might be a sign of pathology.

Present heterogeneity at 10-year follow-up showed a strong tendency to lower VISA-A score, but tendon thickness neither at entry nor at 10-year follow-up correlated with the 10-year VISA-A score, but it seems as if an increased thickness of the tendon is more or less permanent despite change of symptoms and function, as the thickness at entry and at 10-year follow-up correlated.

\section{CONCLUSION}

The very long-term follow-up of patients with AT shows that $90 \%$ report good or excellent results, although many patients $(37 \%)$ still have some degree of pain and reduced function after 10 years. We did not find that one to two CS injections increased the risk of tendon rupture, and with the good or excellent very long-term results CS injections do not seem to have a deleterious effect per se. Patients with insertional tendinopathy were operated more often than mid-substance tendinopathy, but otherwise we found no differences in the long-term outcome between these two groups. We found no prognostic parameters at entry for poor outcome, and it seems that an increased tendon thickness is more or less permanent. Higher grades of flow and heterogeneity seem to correspond with poor function and pain.

Contributors All authors have contributed to this study in preparing the protocol, evaluation of the patients, interpretation of the data and in writing the manuscript.

Funding The primary study was funded by 'Fonden for Faglig udvikling I Speciallægepraksis' governmental support.

Competing interests None declared.

Patient consent Obtained.

Ethics approval According to our local ethical committee (VEK Region Hovedstaden, ethical committee Copenhagen), this study did not need formal approval, as it is considered a quality improvement study.

Provenance and peer review Not commissioned; externally peer reviewed.

Open access This is an open access article distributed in accordance with the Creative Commons Attribution Non Commercial (CC BY-NC 4.0) license, which permits others to distribute, remix, adapt, build upon this work non-commercially, and license their derivative works on different terms, provided the original work is properly cited, appropriate credit is given, any changes made indicated, and the use is non-commercial. See: http://creativecommons.org/licenses/by-nc/4.0/

\section{REFERENCES}

1. Maffulli N, Longo UG, Loppini M, et al. Current treatment options for tendinopathy. Expert Opin Pharmacother 2010;11:2177-86.

2. Silbernagel KG, Thomeé $R$, Thomeé $P$, et al. Eccentric overload training for patients with chronic Achilles tendon pain--a randomised controlled study with reliability testing of the evaluation methods. Scand J Med Sci Sports 2001;11:197-206.

3. Maffulli N, Walley G, Sayana MK, et al. Eccentric calf muscle training in athletic patients with Achilles tendinopathy. Disabil Rehabil 2008;30:1677-84.

4. Ohberg L, Alfredson $\mathrm{H}$. Effects on neovascularisation behind the good results with eccentric training in chronic mid-portion Achilles tendinosis? Knee Surg Sports Traumatol Arthrosc 2004;12:465-70. 
5. Fahlström M, Jonsson P, Lorentzon R, et al. Chronic Achilles tendon pain treated with eccentric calf-muscle training. Knee Surg Sports Traumatol Arthrosc 2003;11:327-33.

6. Nørregaard J, Larsen CC, Bieler T, et al. Eccentric exercise in treatment of Achilles tendinopathy. Scand J Med Sci Sports 2007;17:133-8.

7. Malliaras P, Barton CJ, Reeves ND, et al. Achilles and patellar tendinopathy loading programmes: a systematic review comparing clinical outcomes and identifying potential mechanisms for effectiveness. Sports Med 2013;43:267-86.

8. Beyer R, Kongsgaard M, Hougs Kjær B, et al. Heavy slow resistance versus eccentric training as treatment for achilles tendinopathy: a randomized controlled trial. Am J Sports Med 2015;43:1704-11.

9. Porter D, Barrill E, Oneacre K, et al. The effects of duration and frequency of Achilles tendon stretching on dorsiflexion and outcome in painful heel syndrome: a randomized, blinded, control study. Foot Ankle Int 2002;23:619-24.

10. Fredberg $\mathrm{U}$, Bolvig L, Pfeiffer-Jensen $\mathrm{M}$, et al. Ultrasonography as a tool for diagnosis, guidance of local steroid injection and, together with pressure algometry, monitoring of the treatment of athletes with chronic jumper's knee and Achilles tendinitis: a randomized, double-blind, placebo-controlled study. Scand J Rheumatol 2004;33:94-101.

11. Haynes B. Can it work? Does it work? Is it worth it? The testing of healthcare interventions is evolving. BMJ 1999;319:652-3.

12. Silbernagel KG, Brorsson A, Lundberg $M$. The majority of patients with Achilles tendinopathy recover fully when treated with exercise alone: a 5-year follow-up. Am J Sports Med 2011;39:607-13.

13. van der Plas A, de Jonge $S$, de Vos RJ, et al. A 5-year follow-up study of Alfredson's heel-drop exercise programme in chronic midportion Achilles tendinopathy. Br J Sports Med 2012;46:214-8.

14. Paavola M, Kannus P, Paakkala T, et al. Long-term prognosis of patients with Achilles tendinopathy. Am J Sports Med 2000;28:634-42.

15. Wetke E, Johannsen F, Langberg H. Achilles tendinopathy: a prospective study on the effect of active rehabilitation and steroid injections in a clinical setting. Scand J Med Sci Sports 2015;25:e392-e399.

16. Bakkegaard M, Johannsen FE, Højgaard B, et al. Ultrasonography as a prognostic and objective parameter in Achilles tendinopathy: a prospective observational study. Eur J Radiol 2015;84:458-62.
17. Iversen JV, Bartels EM, Jørgensen JE, et al. Danish VISA-A questionnaire with validation and reliability testing for Danishspeaking Achilles tendinopathy patients. Scand J Med Sci Sports 2016;26:1423-7.

18. Johannsen F, Jensen S, Stallknecht SE, et al. Sonographic measurements of the achilles tendon, plantar fascia, and heel fat pad are reliable: a test-retest intra- and intertester study. J Clin Ultrasound 2016;44:480-6.

19. Coombes BK, Bisset L, Vicenzino B. Efficacy and safety of corticosteroid injections and other injections for management of tendinopathy: a systematic review of randomised controlled trials. Lancet 2010;376:1751-67.

20. Kongsgaard M, Kovanen V, Aagaard P, et al. Corticosteroid injections, eccentric decline squat training and heavy slow resistance training in patellar tendinopathy. Scand J Med Sci Sports 2009;19:790-802.

21. Brinks A, Koes BW, Volkers AC, et al. Adverse effects of extraarticular corticosteroid injections: a systematic review. BMC Musculoskelet Disord 2010;11:206.

22. Jonsson $\mathrm{P}$, Alfredson $\mathrm{H}$, Sunding $\mathrm{K}$, et al. New regimen for eccentric calf-muscle training in patients with chronic insertional Achilles tendinopathy: results of a pilot study. Br J Sports Med 2008;42:746-9

23. Maffulli N, Testa V, Capasso G, et al. Similar histopathological picture in males with Achilles and patellar tendinopathy. Med Sci Sports Exerc 2004;36:1470-5.

24. Reiter M, Ulreich N, Dirisamer A, et al. Colour and power Doppler sonography in symptomatic Achilles tendon disease. Int J Sports Med 2004;25:301-5.

25. De Jonge S, Warnaars JL, De Vos RJ, et al. Relationship between neovascularization and clinical severity in Achilles tendinopathy in 556 paired measurements. Scand J Med Sci Sports 2014;24:773-8.

26. Ohberg L, Alfredson H. Ultrasound guided sclerosis of neovessels in painful chronic Achilles tendinosis: pilot study of a new treatment. $\mathrm{Br}$ J Sports Med 2002;36:173-5

27. Boesen MI, Koenig MJ, Torp-Pedersen S, et al. Tendinopathy and Doppler activity: the vascular response of the Achilles tendon to exercise. Scand J Med Sci Sports 2006;16:463-9. 